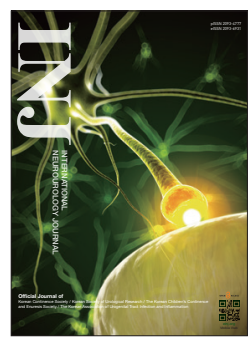

\title{
Extracellular Matrix Revisited: Roles in Tissue Engineering
}

\author{
Youhwan Kim ${ }^{1}$, Hyojin $\mathrm{Ko}^{1}$, Ik Keun Kwon ${ }^{2}$, Kwanwoo Shin ${ }^{1}$ \\ ${ }^{1}$ Department of Chemistry and Institute of Biological Interfaces, Sogang University, Seoul, Korea \\ ${ }^{2}$ Department of Dental Materials, School of Dentistry, Kyung Hee University, Seoul, Korea
}

\begin{abstract}
The extracellular matrix (ECM) is a heterogeneous, connective network composed of fibrous glycoproteins that coordinate in vivo to provide the physical scaffolding, mechanical stability, and biochemical cues necessary for tissue morphogenesis and homeostasis. This review highlights some of the recently raised aspects of the roles of the ECM as related to the fields of biophysics and biomedical engineering. Fundamental aspects of focus include the role of the ECM as a basic cellular structure, for novel spontaneous network formation, as an ideal scaffold in tissue engineering, and its essential contribution to cell sheet technology. As these technologies move from the laboratory to clinical practice, they are bound to shape the vast field of tissue engineering for medical transplantations.
\end{abstract}

Keywords: Extracellular Matrix; Tissue Engineering; Fibronectin; Collagen; Elastin; Cell Sheets

- Grant/Fund Support: This work was supported by the National Research Foundation of Korea via the Korean government (Leading Foreign Research Institute Recruitment Program [2013K1A4A3055268], the Advanced Research Center for Nuclear Excellence [2011-0031932], the Public welfare \& Safety Research Program [HI14C2241], and Mid-career Researcher Program [201100177539]).

- Conflict of Interest: No potential conflict of interest relevant to this article was reported.

\section{INTRODUCTION}

Tissue engineering involves the development of growth and regeneration techniques for connective tissues or organs using a combination of cells and a scaffold to produce a functional organ [1]. These engineering techniques therefore begin with the implantation of artificial materials, providing a proper environment for cells or tissues to be grown and functionalized [2]. The scaffolds are often formulated with a biodegradable polymer, extracellular matrix (ECM), and growth factors, serving as a skeleton to be filled up with cells, and eventually grow into 3-dimensional tissues. Given the importance of intercellular connections in the field of tissue engineering, considerable efforts have been made to design an artificial ECM composed of proteoglycan (PG) and fibrous proteins in vitro. It is particularly important to achieve an architecture of a 3-dimensional network and determine the effective ingredients in its chemical composition. Among the essential noncellular components, the ECM is a heterogeneous, connective network composed of fibrous glycoproteins, PGs, and small molecules that coordinate in vivo to provide the physical scaffolding, mechanical stability, and biochemical cues necessary for tissue morphogenesis and homeostasis.

The overview and importance of the ECM has been wellsummarized in a review by Frantz et al. [3] and references therein, which mainly focuses on the ECM molecular composition, ECM and tissue homeostasis, ECM and tissue aging, tensional homeostasis, and fibrosis, and the challenges related to natural and synthetic engineered ECMs. In this review, we focus on selected aspects of the roles of the ECM that have been recently

Corresponding author: Kwanwoo Shin (iD http://orcid.org/0000-0002-7563-8581 Department of Chemistry and Institute of Biological Interfaces, Sogang University, 35 Baekbeom-ro, Mapo-gu, Seoul 04107, Korea

E-mail: kwshin@sogang.ac.kr / Tel: +82-2-705-8441 / Fax: +82-2-701-0967

Submitted: May 3, 2016 / Accepted after revision: May 14, 2016 
put forth in the fields of biophysics and biomedical engineering, which have not yet been generally addressed, in order to shed light on the most suitable biological ECM materials that can be utilized as key materials in tissue engineering.

\section{BIOMEDICAL CONTEXT OF THE EXTRACELLULAR MATRIX}

\section{Interaction Between the ECM and Cytoskeleton}

One of the most important aspects is to obtain a detailed understanding of how the ECM network interacts with cytoskeletal networks in cells. The cytoskeletal network in most eukaryotic cells is also a combinatorial form of polymeric networks made of actin filaments, microtubules, and intermediate filaments $[4,5]$. These networks play an essential role in determining not only the shape and mechanics of a cell but also, and more importantly, cell motility. In particular, the orchestrated movement of cells in particular directions to specific locations is an essential requirement during natural tissue development [6]. These cellular migrations are often explained using a cytoskeletal model; the spontaneous cycling of the polymerization and depolymerization of cytoskeletal filaments leads to cellular motility at the front of a cell that forms a tight interface with the extracellular network.

In order to understand the cytoskeletal mechanism in a cell, the concept of symmetry breaking was introduced. For example, individual actin filaments and microtubules are structurally and kinetically polarized to generate pushing forces and pulling forces, reflecting the asymmetric nature of the filament organization in cytoskeletal networks [7]. The mechanical properties (i.e., rigidity and elasticity) of the external network are known to influence the polarized organization of cytoskeletal fibers in the target cells. Therefore, development of an optimized ECMbased scaffold, from a simple supporting scaffold to a more complex dynamic bioactive environment, requires consideration that the internal cytoskeletal fibers may respond differently against the given extracellular matrices, both biologically and physically. Hence, the effectiveness of the scaffold in terms of both cellular growth (chemical response) and cellular migration (physical response) should be reassessed.

\section{Spontaneous Formation of ECM Networks}

As described in the Introduction, the ECM is a heterogeneous, connective "network" that is coordinated in vivo to provide a physical scaffolding for cells and tissues [8]. To maintain the structure of the network in nature, polyanionic PGs such as chondroitin sulfate and heparin sulfate, with sulfonic acids as functional groups, coordinate and couple with glycoproteins while stabilizing the tissue mechanics [9-11]. Several attempts have been made to explain how the ECM protein fibrillogenesis is initiated via external mechanical forces or electrostatic surface charges in vitro [12-24]. For example, Ulmer et al. [13] observed that an external shear-force on hydrophobic micropatterned pillars could induce unfolded fibronectin (FN) molecules, driving the formation of an FN network. Feinberg and Parker [25] developed highly ordered FN nanofabrics, and subsequent work suggested that the conformational unfolding of FN fibrils could be induced using microcontact printing onto thermosensitive polymer substrates, and the release of these patterns led to fibrillogenesis [12]. Alternatively, instead of mechanical forces, Pernodet et al. [14] used highly charged polymer surfaces, with charge densities equivalent to or higher than the cell's surface charge $\left(0.10 \mathrm{C} / \mathrm{m}^{2}\right)$, to initiate $\mathrm{FN}$ molecular unfolding and spontaneous fibrillogenesis. More recently, Ballester-Beltran et al. $[15,16]$ showed that exposing FN molecules to specific surface chemistries also induced FN fibril assembly. In contrast to previous results obtained under applied external forces, the surface-triggered FN networks were limited to randomly organized physiological networks [17-19].

Although considerable research effort has been devoted to manufacturing fibers and fabrics composed of fibrous ECM proteins using mechanical or biochemical cues, little is known about how the protein conformation affects fibrillogenesis with respect to the local distributions of PGs. FN, laminin (LAM), and elastin (ELAS) all contain secondary structures rich in $\beta$-strands (accounting for $47 \%, 15 \%$, and $36 \%$ of the total secondary structures, respectively) [26-28], whereas collagen (COL) contains a secondary structure rich in $\alpha$-helices (accounting for $40 \%$ of the total secondary structures) [29,30]. FN and LAM form networks through receptor-triggered self-assembly, whereas ELAS and COL form fibers via cross-linking [31-34]. Moreover, FN and COL are present throughout the body, whereas LAM and ELAS are localized primarily in the basement membrane and connective tissue, respectively [32,3537].

Recently, Ahn et al. [38] developed a method to guide the spontaneous polymerization of ECM glycoproteins along PG mimetic patterns, which were used to recruit, stabilize, and polymerize fibrillar ECM proteins. They showed that this process could effectively mimic the FN assembly model in vivo (Fig. 
a

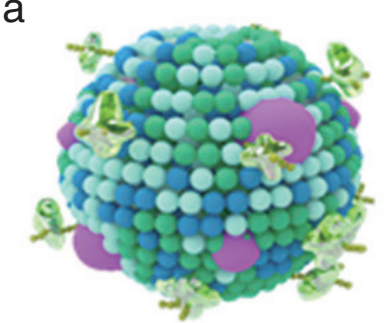

b

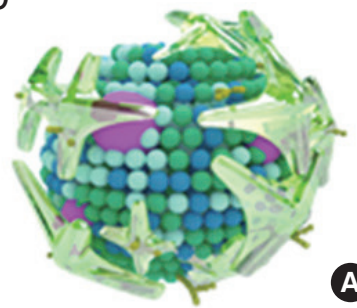

a

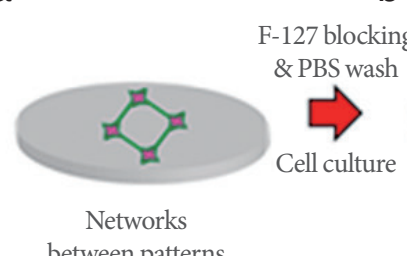

between patterns b

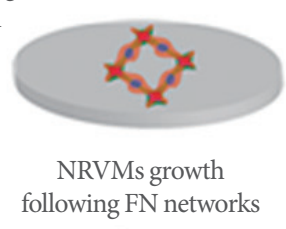

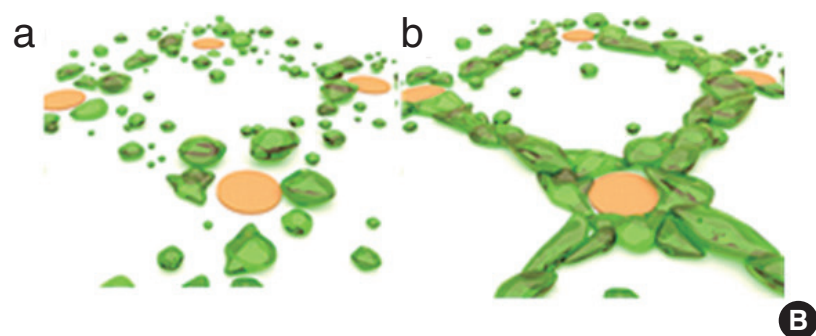
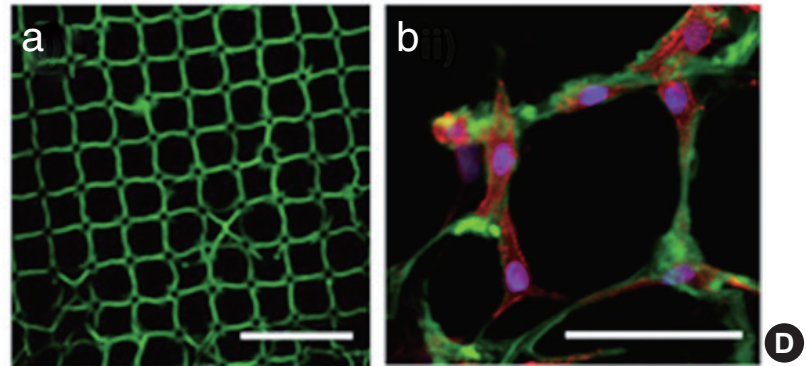

Fig. 1. Spontaneous network formation of fibronectin (FN). (A) Schematic model of FN assembly on a cellular membrane: (a) receptors on the membrane, i.e., integrin, recruit the FN molecules; (b) the unfolded FNs at integrin promote FN-FN interactions and fibril assembly. (B) Spontaneous FN network formation of a proposed mechanistic model on the patterned surface: (a) FN is collected near the charged domains and consecutively propagates networks to (b). (C) Schematics of the FN network cultured with cells through (a) and (b). (D) Confocal microscope images of (a) FN network on polystyrene sulfonated acid islands, scale bar is $100 \mu \mathrm{m}$; (b) FN network (green) cultured with neonatal rat ventricular myocytes (NRVMs), stained for the nucleus (4',6-diamidino-2-phenylindole), aactinin (red). Scale bar is $50 \mu \mathrm{m}$. PBS, phosphate-buffered saline. Adapted from Ahn et al. Adv Mater 2015;27:2838-45 [38].

1A); essentially, binding of FN molecules to integrin induces FN unfolding, thereby spontaneously promoting FN-FN interactions and fibril assembly. Using the FN assembly model, they hypothesized that FN on the receptor-like domain's pattern can self-assemble into networks, and found that these precisely controlled ECM connections provide a flexible, transferable substrate that can be customized for use as tissue-engineered cardiac scaffolds (Fig. 1B and C). First, FN binding to highly charged domains (polystyrene sulfonated acids, PSS) induces protein unfolding due to a strong electrostatic interaction with the sulfonic groups exposed from the PSS. Second, an increase in the concentration of FN near the unfolded FN at the PSS spots leads to molecular assembly, which forms a cluster of fibrillated FN molecules and becomes a node for the FN network. Third, once a fibril is formed, other FN molecules in the FN solution are sequentially connected to it in a process of energy-dependent nucleation. Finally, during the postincubation step, the adsorbed FN film is ruptured at the substrate and connected to the fibrillated FN domains. As shown in Fig. 1D, the in vivo-like FN networks were successfully generated. In addition, cells could be grown and aligned through the PG mimetics-induced FN networks, thus demonstrating the biocompatibility of the networks (Fig. 1D). Neonatal rat ventricular myocytes selectively contracted in response to topological cues from the networks, whereas the cardiac cells on isotropic FN surfaces were found to be randomly distributed. The interplay between the hanging networks and cardiac cells revealed that a concurrent contraction with a minimal anchoring junction serves as an efficient cell patch system [38]. These networks can be released from the substrate as films and then used as substrates for the cultures of various primary cultured cells. Thus, further attempts to mimic the assembly of the native ECM may provide insights into the assembly of mechanically and biochemically robust fibers for applications in tissue engineering.

\section{ECM as a Native Scaffold}

Tissue engineering relies on the proper use of a combination of cells, scaffolds, and cell-growth stimuli factors to effectively reconstruct damaged tissues for regenerative wound-healing purposes [39]. In general, tissue engineering requires a scaffold, which is typically made of engineered biocompatible polymers or naturally existing ECM proteins, providing the structural support for cell attachment and subsequent tissue development. Since most cells in nature develop into tissues while residing in 
a matrix, the scaffold used in tissue engineering applications must have the following functions $[1,2]$ :

(1) Structural support for cells: cells must adhere onto a solid surface, and grow, migrate, and proliferate until they function normally in a manner similar to the surrounding tissues and organs.

(2) Provide a mechanical environment to cells: scaffolds have to provide adequate mechanical properties, similar to the matrix native to the implanted sites. Depending on the anatomical sites, the mechanical properties, i.e. toughness, rigidity, and elasticity, will vary for the required functions.

(3) Biodegradability for the formation of functional microvascular networks and remodeling: in practice, the role of the scaffold is temporary, and is required only until the patient's own ECM can replace the implanted structures. In addition, the degradable scaffolds provide room, over time, for neovascularization and remodeling in response to tissue formation.

(4) Biocompatibility and bioactivities: engineered scaffolds often induce a foreign body reaction, resulting severe inflammation due to immunologic responses; therefore, a scaffold must induce a negligible immune reaction to the residing cells. More actively, the scaffolds may provide bioactive cues and growth factors to regulate the cellular activities.

Unfortunately, the use of engineered scaffolds made of nonnative materials often fails to produce intact cell-cell junctions owing to the unnatural cellular textures, thereby isolating the cell-bunches from the surroundings [40,41]. To overcome this challenge, instead of biodegradable polymeric scaffolds, the use of ECM in native tissues or scaffolds synthesized from natural ECM proteins can provide the ideal features listed above in engineered tissues. For example, COL, which is the most common protein in the body, can provide adequate mechanical rigidity and structural stability, depending on the bundling thickness and directional order $[36,42]$. ELAS is highly elastic, being responsible for the stretching or contracting of tissues, and is thus often used to reinforce the mechanical properties of COL-based scaffolds $[24,37,43]$. LAM is a major component of the basal lamina, influencing cell differentiation, migration, and adhesion $[23,32,44]$. FN is responsible for the binding of other ECM components such as COL, fibrin, and heparin sulfate, as previously reviewed $[11,17,18,31,35]$. There are numerous types of ECM in tissues, and a specific tissue is composed of multiple ECM components. Hence, the ideal scaffold for regenerative medicine will be one with the ECM architecture of the target tissue in its native state; however, accurately mimicking the native ECM for engineered tissues remains a challenge given the current technology [8].

\section{Challenges in Tissue Engineering: Cell Sheet Technology}

Most approaches used in regenerative medicine can be generally categorized into 3 techniques: (1) autotransplantation, which is the transplantation of proteins, cells, or tissues (so-called autografts) from one part of the body (i.e., the tooth [45] or heart [46]) to another in the same patient; (2) allotransplantation, which is the transplantation of cells, tissues, or organs (so-called allografts) to a recipient from genetically nonidentical donors of the same species [47,48]; and (3) xenotransplantation, which is the transplantation of living cells, tissues, or organs (so-called xenografts) from one species to another [49,50]. In addition to ethical issues [51], significant potential problems have limited the use of transplantations with allografts and xenografts for medical treatments, including immunologic responses, xenozoonosis, and genetic alterations [52]. Hence, achieving wound healing via autografts remains an ideal, yet challenging approach [53].

As an alternative approach, Yamato and Okano [54] developed the field of so-called "cell sheet engineering," which enables the formation of multiple single-cell layers without requiring any polymeric scaffolds. Cells cultured on a sacrificial layer secrete ECM, forming tight cell-cell junctions. A confluent cell layer is detached and then overlaid on top of another confluent cell layer, resulting in a thicker tissue-like architecture after repeated processes, such as the cornea [55,56], cardiac muscles [57,58], liver lobule, and kidney glomeruli [59]. They named these architectures as cell sheets, which have now been applied in various forms in several clinical trials. Based on their clinical application, this cell sheet engineering approach appears to be a feasible option for achieving scaffold-free tissue formation [60]. Numerous reports have shown the successful use of multilayered corneal epithelial cell sheet transplantation and regeneration, periodontal ligament cell sheet transplantation and regeneration, and bladder augmentation with urothelial cell sheets [61]. A recent report further hinted at the possibility of using cell sheets for 3-dimensional heart tissue formation to create a cardiac patch to repair impaired hearts [62]. It has been noted that one of the major advantages of cell sheet technology is the use of the native ECM between the single cell sheets, providing intact cell-cell junctions. Ironically, one of the disadvantages of this method is that constructing ECM-rich tissues from cells showing limited 
ECM secretion can be difficult in some contexts.

\section{SUMMARY AND PERSPECTIVES}

The ECM provides the physical and functional microenvironment in which cells exist. Therefore, the aim of scaffold design in engineered tissues is to closely mimic the ECM architecture of target tissues as much as possible, with respect to both structure and function. Fundamentally, researchers have been able to understand the unique roles of the ECM in tissue growth and regeneration, and have used this knowledge to realize the design of artificial ECMs and develop associated techniques and materials to implement their use. As these technologies move from the laboratory to clinical practice, they are bound to shape the vast field of tissue engineering for medical transplantations. Given the positive impact of these technologies on the quality of life of patients, ECM-based regenerative technology should be continuously reassessed and explored from both a perspective of gaining fundamental understanding and in clinical application.

\section{REFERENCES}

1. Brien FJ. Biomaterials \& scaffolds for tissue engineering. Mater Today 2011;14:88-95.

2. Chan BP, Leong KW. Scaffolding in tissue engineering: general approaches and tissue-specific considerations. Eur Spine J 2008;17 Suppl 4:467-79.

3. Frantz C, Stewart KM, Weaver VM. The extracellular matrix at a glance. J Cell Sci 2010;123(Pt 24):4195-200.

4. Mullins RD. Cytoskeletal mechanisms for breaking cellular symmetry. Cold Spring Harb Perspect Biol 2010;2:a003392.

5. Ridley AJ, Hall A. The small GTP-binding protein rho regulates the assembly of focal adhesions and actin stress fibers in response to growth factors. Cell 1992;70:389-99.

6. Gumbiner BM. Cell adhesion: the molecular basis of tissue architecture and morphogenesis. Cell 1996;84:345-57.

7. Wierzbicka-Patynowski I, Schwarzbauer JE. The ins and outs of fibronectin matrix assembly. J Cell Sci 2003;116(Pt 16):3269-76.

8. Rosales AM, Anseth KS. The design of reversible hydrogels to capture extracellular matrix dynamics. Nat Rev Mat 2016;1:15012.

9. Schaefer L, Schaefer RM. Proteoglycans: from structural compounds to signaling molecules. Cell Tissue Res 2010;339:237-46.

10. Rozario T, DeSimone DW. The extracellular matrix in development and morphogenesis: a dynamic view. Dev Biol 2010;341:126-40.
11. Deravi LF, Golecki HM, Parker KK. Protein-based textiles: bio-inspired and bio-derived materials for medical and non-medical applications. J Chem Biol Interface 2013;1:25-34.

12. Deravi LF, Su T, Paten JA, Ruberti JW, Bertoldi K, Parker KK. Differential contributions of conformation extension and domain unfolding to properties of fibronectin nanotextiles. Nano Lett 2012; 12:5587-92.

13. Ulmer J, Geiger B, Spatz JP. Force-induced fibronectin fibrillogenesis in vitro. Soft Matter 2008;4:1998-2007.

14. Pernodet N, Rafailovich M, Sokolov J, Xu D, Yang NL, McLeod K. Fibronectin fibrillogenesis on sulfonated polystyrene surfaces. J Biomed Mater Res A 2003;64:684-92.

15. Ballester-Beltrn J, Cantini M, Lebourg M, Rico P, Moratal D, Garcia AJ, et al. Effect of topological cues on material-driven fibronectin fibrillogenesis and cell differentiation. J Mater Sci Mater Med 2012;23:195-204.

16. Ballester-Beltrn J. Rico P, Moratal D, Song W, Mano JF, SalmeronSánchez M. Role of superhydrophobicity in the biological activity of fibronectin at the cell-material interface. Soft Matter 2011; : 10803-11.

17. Salmeron-Sanchez M, Rico P, Moratal D, Lee TT, Schwarzbauer JE, García AJ. Role of material-driven fibronectin fibrillogenesis in cell differentiation. Biomaterials 2011;32:2099-105.

18. Gugutkov D, Gonzalez-Garcia C, Rodriguez Hernandez JC, Altankov G, Salmeron-Sanchez M. Biological activity of the substrateinduced fibronectin network: insight into the third dimension through electrospun fibers. Langmuir 2009;25:10893-900.

19. Nelea V, Kaartinen MT. Periodic beaded-filament assembly of fibronectin on negatively charged surface. J Struct Biol 2010;170:509.

20. Freire E, Coelho-Sampaio T. Self-assembly of laminin induced by acidic pH. J Biol Chem 2000;275:817-22.

21. Matthews JA, Wnek GE, Simpson DG, Bowlin GL. Electrospinning of collagen nanofibers. Biomacromolecules 2002;3:232-8.

22. Li Y, Asadi A, Monroe MR, Douglas EP. pH effects on collagen fibrillogenesis in vitro: electrostatic interactions and phosphate binding. Mater Sci Eng C Mater Biol Appl 2009;29:1643-9.

23. Neal RA, McClugage SG, Link MC, Sefcik LS, Ogle RC, Botchwey EA. Laminin nanofiber meshes that mimic morphological properties and bioactivity of basement membranes. Tissue Eng Part C Methods 2009;15:11-21.

24. Buttafoco L, Kolkman NG, Engbers-Buijtenhuijs P, Poot AA, Dijkstra PJ, Vermes I, et al. Electrospinning of collagen and elastin for tissue engineering applications. Biomaterials 2006;27:724-34.

25. Feinberg AW, Parker KK. Surface-initiated assembly of protein 
nanofabrics. Nano Lett 2010;10:2184-91.

26. Pauthe E, Pelta J, Patel S, Lairez D, Goubard F. Temperature-induced beta-aggregation of fibronectin in aqueous solution. Biochim Biophys Acta 2002;1597:12-21.

27. Ott U, Odermatt E, Engel J, Furthmayr H, Timpl R. Protease resistance and conformation of laminin. Eur J Biochem 1982;123:63-72.

28. Debelle L, Alix AJ, Wei SM, Jacob MP, Huvenne JP, Berjot M, et al. The secondary structure and architecture of human elastin. Eur J Biochem 1998;258:533-9.

29. Petibois C, Gouspillou G, Wehbe K, Delage JP, Deleris G. Analysis of type I and IV collagens by FT-IR spectroscopy and imaging for a molecular investigation of skeletal muscle connective tissue. Anal Bioanal Chem 2006;386:1961-6.

30. Petibois C, Déléris G. Chemical mapping of tumor progression by FT-IR imaging: towards molecular histopathology. Trends Biotechnol 2006;24:455-62.

31. Singh P, Carraher C, Schwarzbauer JE. Assembly of fibronectin extracellular matrix. Annu Rev Cell Dev Biol 2010;26:397-419.

32. Colognato H, Winkelmann DA, Yurchenco PD. Laminin polymerization induces a receptor-cytoskeleton network. J Cell Biol 1999; 145:619-31.

33. Kadler KE, Hill A, Canty-Laird EG. Collagen fibrillogenesis: fibronectin, integrins, and minor collagens as organizers and nucleators. Curr Opin Cell Biol 2008;20:495-501.

34. Reiser K, McCormick RJ, Rucker RB. Enzymatic and nonenzymatic cross-linking of collagen and elastin. FASEB J 1992;6:2439-49.

35. Schwarzbauer JE, DeSimone DW. Fibronectins, their fibrillogenesis, and in vivo functions. Cold Spring Harb Perspect Biol 2011; 3:a005041.

36. Kadler KE, Baldock C, Bella J, Boot-Handford RP. Collagens at a glance. J Cell Sci 2007;120(Pt 12):1955-8.

37. Debelle L, Tamburro AM. Elastin: molecular description and function. Int J Biochem Cell Biol 1999;31:261-72.

38. Ahn S, Deravi LF, Park SJ, Dabiri BE, Kim JS, Parker KK, et al. Selforganizing large-scale extracellular-matrix protein networks. Adv Mater 2015;27:2838-45.

39. Ko IK, Lee SJ, Atala A, Yoo JJ. In situ tissue regeneration through host stem cell recruitment. Exp Mol Med 2013;45:e57.

40. Anderson JM, Rodriguez A, Chang DT. Foreign body reaction to biomaterials. Semin Immunol 2008;20:86-100.

41. Yang PJ, Temenoff JS. Engineering orthopedic tissue interfaces. Tissue Eng Part B Rev 2009;15:127-41.

42. Glowacki J, Mizuno S. Collagen scaffolds for tissue engineering. Biopolymers 2008;89:338-44.

43. Berglund JD, Nerem RM, Sambanis A. Incorporation of intact elastin scaffolds in tissue-engineered collagen-based vascular grafts. Tissue Eng 2004;10:1526-35.

44. Tate CC, Shear DA, Tate MC, Archer DR, Stein DG, LaPlaca MC. Laminin and fibronectin scaffolds enhance neural stem cell transplantation into the injured brain. J Tissue Eng Regen Med 2009;3: 208-17.

45. Kvint S, Lindsten R, Magnusson A, Nilsson P, Bjerklin K. Autotransplantation of teeth in 215 patients: a follow-up study. Angle Orthod 2010;80:446-51.

46. Conklin LD, Reardon MJ. Autotransplantation of the heart for primary cardiac malignancy: development and surgical technique. Tex Heart Inst J 2002;29:105-8.

47. Bueno EM, Diaz-Siso JR, Sisk GC, Chandawarkar A, Kiwanuka H, Lamparello B, et al. Vascularized composite allotransplantation and tissue engineering. J Craniofac Surg 2013;24:256-63.

48. Diaz-Siso JR, Bueno EM, Sisk GC, Marty FM, Pomahac B, Tullius SG. Vascularized composite tissue allotransplantation: state of the art. Clin Transplant 2013;27:330-7.

49. Chatterjee DS. A controlled comparative study of the use of porcine xenograft in the treatment of partial thickness skin loss in an occupational health centre. Curr Med Res Opin 1978;5:726-33.

50. Michler RE. Xenotransplantation: risks, clinical potential, and future prospects. Emerg Infect Dis 1996;2:64-70.

51. Sobbrio P, Jorqui M. An overview of the role of society and risk in xenotransplantation. Xenotransplantation 2014;21:523-32.

52. Michaels M. Xenozoonoses and the xenotransplant recipient. Ann N Y Acad Sci 1998;862:100-4.

53. Hoffman MD, Benoit DS. Emerging ideas: Engineering the periosteum: revitalizing allografts by mimicking autograft healing. Clin Orthop Relat Res 2013;471:721-6.

54. Yamato M, Okano T. Cell sheet engineering. Mater Today 2004; 7;42-7.

55. Oie Y, Nishida K. Regenerative medicine for the cornea. Biomed Res Int 2013;2013:428247.

56. Nishida K, Yamato M, Hayashida Y, Watanabe K, Yamamoto K, Adachi E, et al. Corneal reconstruction with tissue-engineered cell sheets composed of autologous oral mucosal epithelium. N Engl J Med 2004;351:1187-96.

57. Shimizu T, Yamato M, Akutsu T, Shibata T, Isoi Y, Kikuchi A, et al. Electrically communicating three-dimensional cardiac tissue mimic fabricated by layered cultured cardiomyocyte sheets. J Biomed Mater Res 2002;60:110-7.

58. Masuda S, Shimizu T, Yamato M, Okano T. Cell sheet engineering for heart tissue repair. Adv Drug Deliv Rev 2008;60:277-85.

59. Yang J, Yamato M, Shimizu T, Sekine H, Ohashi K, Kanzaki M, et 
al. Reconstruction of functional tissues with cell sheet engineering. Biomaterials 2007;28:5033-43.

60. Yang J, Yamato M, Kohno C, Nishimoto A, Sekine H, Fukai F, et al. Cell sheet engineering: recreating tissues without biodegradable scaffolds. Biomaterials 2005;26:6415-22.
61. Wang J, Zhang R, Shen Y, Xu C, Qi S, Lu L, et al. Recent advances in cell sheet technology for periodontal regeneration. Curr Stem Cell Res Ther 2014;9:162-73.

62. Hirt MN, Hansen A, Eschenhagen T. Cardiac tissue engineering: state of the art. Circ Res 2014;114:354-67. 